\title{
The solar-interior equation of state with the path-integral formalism
}

\section{Domain of validity}

\author{
A. Perez ${ }^{1,2}$, K. Mussack ${ }^{3,4}$, W. Däppen ${ }^{3}$, and D. $\mathrm{Mao}^{3}$ \\ ${ }^{1}$ Laboratoire de Physique Théorique (UMR CNRS/ULP 7085), Université Louis Pasteur de Strasbourg, 67084 Strasbourg Cedex, \\ France \\ e-mail: perez@lpt1.u-strasbg.fr \\ 2 Department of Applied Physics, Jerusalem College of Technology, 92221 Jerusalem, Israel \\ ${ }^{3}$ Department of Physics and Astronomy, USC, Los Angeles, CA 90089-1342, USA \\ e-mail: [dappen;dmao]@usc.edu \\ 4 Institute of Astronomy, University of Cambridge, Cambridge CB3 OHA, UK \\ e-mail: mussack@ast.cam.ac.uk
}

Received 25 April 2007 / Accepted 19 July 2009

\section{ABSTRACT}

\begin{abstract}
Aims. This is the first paper in a series that deals with solar-physics applications of the equation-of-state formalism based on the formulation of the so-called "Feynman-Kac (FK) representation". Here, the FK equation of state is presented and adapted for solar applications. Its domain of validity is assessed. The practical application to the Sun will be dealt with in Paper II. Paper III will extend the current FK formalism to a higher order.

Methods. A recent rigorous quantum-statistical formalism for Coulomb systems is used to compute the thermodynamical quantities for solar modeling, taking into account the necessary requirements on smoothness and accuracy. The FK formalism being a virial expansion, it suffers from the well-known deficiency that it is limited to nearly full ionization. This point is elaborated in detail, and the quantitative criterion for the domain of validity of the FK equation of state is established.

Results. Use of the FK equation of state is limited to physical conditions for which more than $90 \%$ of helium is ionized. This includes the inner region of the Sun out to about .98 of the solar radius. Despite this limitation, in the parts of the Sun where it is applicable, the FK equation of state has the power to be more accurate than the equations of state currently used in solar modeling. The FK approach is especially suited to study physical effects such as Coulomb screening, bound states, the onset of recombination of fully ionized species, as well as diffraction and exchange effects.

Conclusions. Despite technical difficulties in its application, there are unique features in the FK approach that promise to turn it into the most exact of the available formalisms, provided FK is restricted to the deeper layers of the Sun where more than $90 \%$ of helium is ionized. The localizing power of helioseismology allows a test of the FK equation of state. Such a test will be beneficial both for better solar models and for tighter solar constraints of the equation of state.
\end{abstract}

Key words. equation of state - stars: interiors - Sun: interior - Sun: helioseismology

\section{Introduction}

This paper is the first of a series of three devoted to the study of the equation of state based on the formalism of the path integral in the framework of the Feynman-Kac (hereinafter FK) representation. This formalism leads to a virial expansion of the thermodynamic functions in powers of the total density of a Coulomb plasma (Alastuey \& Perez 1992, 1996; Alastuey et al. 1994, 1995). The path-integral approach is the latest in a series of attempts to improve the equation of state for the solar interiors. However, so far there has only been a very limited amount of astrophysical applications of that formalism. One (Perez \& Chabrier 2004) was an application to the screening enhancement of nuclear fusion. Another one was a precursor (Perez \& Däppen 1995) of the present series of articles, which addressed the possibility of using the FK formalism in solar and stellar modeling. Here, we embark on a more systematic application of the FK representation. It is well known that solar and stellar modeling requires thermodynamic quantities that are smooth, consistent, valid over a large range of temperatures and densities, and incorporate the most important astrophysically relevant chemical elements. Let us briefly review the two major equation-of-state efforts of the last 20 years. Both were made in the context of the two most recent opacity recalculations.
One of them is the international Opacity Project (OP; see the books by Seaton 1995; Berrington 1997); it contains the socalled Mihalas-Hummer-Däppen equation of state (Däppen et al. 1988; Hummer \& Mihalas 1988; Mihalas et al. 1988; Nayfonov et al. 1999; Trampedach et al. 2006, hereinafter MHD), dealing with heuristic concepts about the modification of atoms and ions in a plasma. Specifically, in MHD the destruction of atomic states is modeled, on the one hand by neutral species according to excluded volume, and on the other hand by Stark dissolution due to the surrounding microfield. From the included 15 astrophysically relevant elements $(\mathrm{H}, \mathrm{He}, \mathrm{C}, \mathrm{N}, \mathrm{O}, \mathrm{Ne}, \mathrm{Na}, \mathrm{Mg}$, $\mathrm{Al}, \mathrm{Si}, \mathrm{S}, \mathrm{Ar}, \mathrm{K}, \mathrm{Ca}, \mathrm{Fe}$ ), users can select any subset. Although MHD in its current version does not contain free parameters, several of the physical quantities are, in principle, open to revision. Possible candidates for adjustment are i) the atomic and ionic radii (which can be chosen to match observations), ii) the strength of the assumed microfield distribution, and iii) the specific form of the interaction potential between species. Since MHD is basically a heuristic equation of state, such future improvements will be legitimate and they can be developed under the guidance of observations. For more details see Trampedach et al. (2006). 
The other recent opacity effort is the OPAL project pursued at Livermore. In contrast to OP, its underlying equation of state has relied on the so-called physical picture, which is built on the notion of fundamental particles only, that is, electrons and nuclei. It provides a systematic method to include non-ideal effects. Its so-called ACTEX ("activity expansion") equation of state (see Rogers 1986; Rogers et al. 1996; Rogers \& Nayfonov 2002, and references therein) became part of the OPAL opacity project at Livermore (Iglesias et al. 1987; Iglesias \& Rogers 1991, 1993, 1995, 1996). For this reason, the ACTEX equation of state is hereinafter referred to as the OPAL equation of state. As mentioned, OPAL is a physical-picture formalism, starting out from the grand-canonical ensemble of a system of the basic constituents (electrons and nuclei) interacting through the Coulomb potential. In the currently released version, the OPAL equation of state includes the following astrophysically relevant elements: $\mathrm{H}$, $\mathrm{He}, \mathrm{C}, \mathrm{N}, \mathrm{O}, \mathrm{Ne}$ (but the OPAL opacity calculation includes other elements, such as Fe, as well). In OPAL, any effects of the plasma environment on the internal states are obtained directly from the statistical-mechanical analysis, rather than by assertion as in the chemical picture. Therefore, in contrast to the intuitive MHD, in OPAL there are no adjustable parameters. On the one hand, this is its strength, on the other hand, it means that one cannot easily upgrade OPAL even if observations were to suggest so. Thus in the current OPAL equation of state tables, any residual inaccuracy is either structural, a result of i) the finite truncation in the underlying activity expansion, and ii) the utilization of effective atomic and ionic potentials, or it could be due to the limited number of chemical elements in the current tables.

The equation of state for solar and stellar structure and seismology has to be formally precise and consistent, even before the question of the accuracy of the physical description is asked. It has to satisfy four conditions: i) a large domain of applicability (in $\rho, T$ ), ii) a high precision of its numerical realization, iii) consistency between the thermodynamic quantities, and iv) the possibility to take into account relatively complex mixtures with at least several of the more abundant chemical elements. These requirement are generally easy to satisfy with relatively simple formalisms, such as the popular, widely used CEFF equation of state (Christensen-Dalsgaard \& Däppen 1992), which is an offspring of the widely used Eggleton-Faulkner-Flannery (EFF) equation of state (Eggleton, Faulkner, \& Flannery 1973), or the other offspring of EFF, the SIREFF equation of state (Rogers et al. 1996).

However, the demands from helioseismology are such that formal considerations are not sufficient. The equation of state has to be accurate, not just smooth. Even state-of-the-art equations of state such as OPAL and MHD are not completely sufficient (Däppen 2006), and improvements are still necessary. Improvements can be made either formally (phenomenologically) or rigorously. A formal option is to merge the good features of OPAL and MHD (Liang \& Däppen 2003; Starostin et al. 2003; Liang 2004; Däppen \& Mao 2009) to create an equation of state that matches reality as closely as possible. However, as a phenomenological effort, such a hybrid formalism would not be a scientific improvement but merely a better computational tool for solar and stellar modeling. As far as the development of a real theory is concerned, genuine improvements in the physical model must be made. The rigorous equation of state in the FK representation is such an improvement. In principle, it is a better theory than either MHD or OPAL. A case in point is the treatment of Coulomb screening. Here, the electrons are taken into account with the exact quantum-mechanical exchange and diffraction terms. These are corrections that deal, on the one hand, with the "Pauli blocking" of electrons (exchange terms) and, on the other hand, their finite thermal de Broglie wavelength (diffraction terms). For these terms, both MHD and OPAL use approximations. However, this better physics of FK comes with a price: the FK equation of state has a limited domain of validity unlike the more global MHD and OPAL. It only works for nearly fully ionized plasmas.

As a theory, FK is quite similar to OPAL, since it is realized in the physical picture and the grand-canonical ensemble. However, its net result is that of a virial (density) expansion (unlike OPAL, which is based on activity). The virial coefficients of the FK expansion are evaluated by the path-integral formalism, which in its essence uses the equivalence between a point-charge quantum system and a classical one made of extended filaments. In this spirit, the calculation of all non-ideal contributions to the equation of state becomes possible, systematically, exactly, and analytically. The fact that FK has the form of a virial expansion is the source of its limitation to near full ionization. In Sect. 2 we illustrate the well-known fact that in the case of reacting gases, virial expansions are inadequate to deal with recombination (unlike the activity expansions of OPAL). We discuss this deficiency quite in detail and develop a quantitative criterion for the validity of the FK equation of state. The adopted criterion is a limitation to physical conditions such that more than $90 \%$ of helium is ionized. The subsequent sections discuss the physical condition quantitatively, in terms of a solar model.

Despite the aforementioned limitation to nearly fully ionized matter, the FK approach can be applied to solar modeling. The reason for this is twofold. First, a large part of the solar interior (out to about .98 of the solar radius) lies inside its domain of validity, and second, helioseismology allows a localized analysis of thermodynamic properties despite the fact that there is no complete coverage of the Sun by the FK equation of state.

Here the FK equation of state is presented and its domain of validity discussed. Paper II will then be dedicated to solar applications. They will be based on "mixed" thermodynamic tables, with FK in the highly ionized part, and a conventional equation of state (OPAL or MHD) outside the domain of validity of FK. We note in passing that once these tables exist they can be used also for stellar modeling, but it is not clear if stellar application would ever require such an accuracy of the equation of state, in contrast to solar application for which this need has been well established (see, e.g. Däppen 2006). In any case, stellar applications are beyond the scope of this current series of papers. Finally, paper III will be a natural extension of the current level of the FK formalism. While the current level of FK is based on an expansion of pressure in terms of the density with up to order $\rho^{5 / 2}$, the next (rather elaborate) step is the extension to calculation of the thermodynamic functions up to order $\rho^{3}$. Then we will be able to take into account three-body effects, allowing us to treat helium exactly, beyond the hydrogenic approximation.

\section{Illustration of the limitation of virial expansions for reacting systems}

The principle can be understood by considering the exact equation of the ideal (non-relativistic) Fermi gas. One starts out from an implicit equation of state, obtained from the grand-canonical partition function (see, e.g. Huang 1963)

$\frac{p}{k T}=\frac{1}{\lambda^{3}} f_{5 / 2}(\tilde{z}), \quad \frac{N}{V}=\frac{1}{\lambda^{3}} f_{3 / 2}(\tilde{z})$.

Here, $p, T, N, V, k, \lambda$ are pressure, temperature, particle number, volume, Boltzmann constant, and the thermal de Broglie length 
$\lambda=h / \sqrt{2 \pi m k T}$, respectively. The fugacity $\tilde{z}=\exp (\mu /(k T))(\mu$ being the chemical potential) is defined implicitly by Eq. (1), by virtue of the Fermi integrals

$f_{5 / 2}(\tilde{z})=\frac{4}{\sqrt{\pi}} \int_{0}^{\infty} \mathrm{d} x x^{2} \ln \left(1+\tilde{z} \mathrm{e}^{-x^{2}}\right)$,

$f_{3 / 2}(\tilde{z})=\tilde{z} \frac{\mathrm{d}}{\mathrm{d} \tilde{z}} f_{5 / 2}(\tilde{z})$.

Expanding the Fermi integrals as a series in the high-temperature limit, and eliminating the fugacity $\tilde{z}$, one obtains the virial (density) expansion

$\frac{p V}{N k T}=1+\frac{\lambda^{3}}{2^{5 / 2}} \frac{N}{V}+O\left(\frac{N^{2}}{V^{2}}\right)+\cdots$.

Alternatively, one can continue to work with fugacity expansion

$$
\begin{aligned}
& \frac{p}{k T}=\frac{1}{\lambda^{3}}\left[\tilde{z}-\frac{1}{2^{5 / 2}} \tilde{z}^{2}+\cdots\right], \\
& \frac{N}{V}=\frac{1}{\lambda^{3}}\left[\tilde{z}-\frac{1}{2^{3 / 2}} \tilde{z}^{2}+\cdots\right] .
\end{aligned}
$$

With the introduction of the activity $z=\tilde{z} / \lambda^{3}$ we obtain an even more convenient expansion

$$
\begin{aligned}
& \frac{p}{k T}=z-\frac{\lambda^{3}}{2^{5 / 2}} z^{2}+\cdots, \\
& \frac{N}{V}=z-\frac{\lambda^{3}}{2^{3 / 2}} z^{2}+\cdots .
\end{aligned}
$$

These expressions above are exact for the partially degenerate ideal Fermi gas, they retain their formal structure for any real gas (this follows from their origin in the grand-canonical partition function). Therefore, all the physics lies in the value of the coefficients of the expansions. For the activity, one formally writes

$\frac{p}{k T}=z-b_{2} z^{2}+\cdots$,

$\frac{N}{V}=z-2 b_{2} z^{2}+\cdots$,

and for density,

$\frac{p V}{N k T}=1+B_{2} \frac{N}{V}+O\left(\frac{N^{2}}{V^{2}}\right)+\cdots$.

Inspection of Eqs. (4) and (7) reveals the relation

$B_{2}=-b_{2}$.

While we derived the relation for the ideal Fermi gas, Eq. (12) is a general result, valid for all real gases (see, e.g. Huang 1963). The second-virial coefficient is a function of temperature only; an explicit expression is obtained from classical statistical mechanics by the two-body configuration integral over the interaction potential $U_{12}$

$B_{2}=\frac{1}{2} \int\left(1-\mathrm{e}^{-U_{12} /(k T)}\right) \mathrm{d} V$.

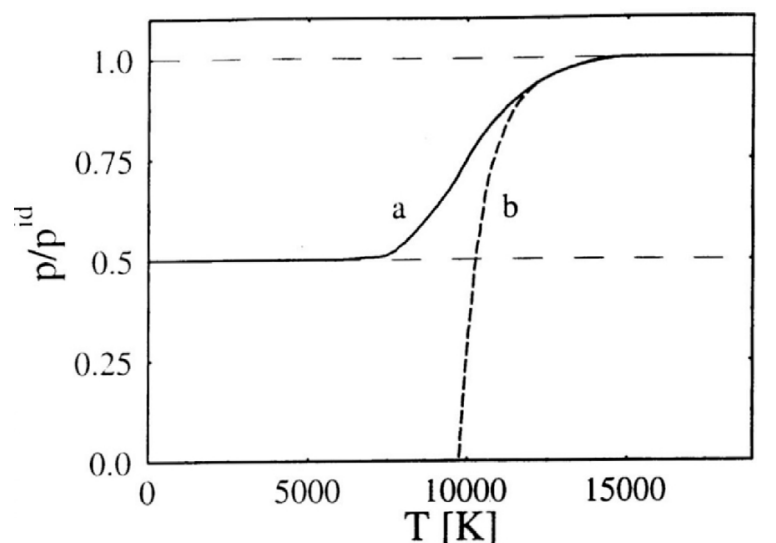

Fig. 1. Illustration of the behavior of activity (solid line) and density expansions (dashed line) for reacting systems, here for the simple reaction of neutral hydrogen $\mathrm{H}$ recombining to hydrogen molecules $\mathrm{H}_{2}$. $P_{\text {id }}$ refers to fully dissociated hydrogen. The activity expansion (a) correctly describes recombination with just one non-ideal term (Eqs. (9) and (10)). Without further high-order terms, the corresponding virial expansion (b) (i.e. the reacting analog to the single-species Eq. (11)) exhibits catastrophic non-physical behavior (negative pressure!) (Ebeling et al. 1976). The case of ionization is analogous.

Of course, as infinite series, activity and density expansions would be strictly equivalent, but when dealing with recombination, a density expansion would have to go to a very high order to match the accuracy of even a second-order activity expansion. Indeed, for recombinations, the activity expansion $(9,10)$ is vastly superior to the virial expansion (11). This is easily seen from Eq. (13), where for a (necessarily) attractive twobody potential and low temperatures $B_{2}$ becomes large negative, which will cause inevitably a negative total pressure at some low temperature. In other words, the virial expansion breaks down. This breakdown is illustrated by the dashed line of Fig. 1 (from Ebeling et al. 1976). For simplicity, that figure is for hydrogen molecular dissociation, but qualitatively it would be the same for ionization. In contrast, the activity expansion can avoid this breakdown, as revealed by a more detailed discussion of Eqs. (9) and (10) that can be found elsewhere (e.g. Ebeling et al. 1976; Kremp et al. 2005). Importantly, already to lowest non-ideal order, the activity expansion can correctly describe complete lowtemperature recombination (solid line in Fig. 1). This property of activity expansions is the principal reason for the remarkable success of the OPAL equation of state.

In contrast, as a virial expansion, the FK equation of state suffers from the pathology illustrated by Fig. 1, irrespective of the fact that its low-order virial coefficients are exact. Nevertheless there is a domain of applicability where we can use FK: Fig. 1 shows the overlap region of the virial and activity expansions. A natural quantitative limit for the application of a virial expansion becomes apparent: as long as the physical conditions are such that more than about $90 \%$ of the bound particles remain ionized, the resulting thermodynamic quantities still will be reliable. In the following we adopt this criterion for the FK approach.

\section{The path-integral formalism in the framework of the Feynman-Kac representation}

In many situations, stellar matter can be adequately described in terms of quantum plasmas made of electrons and nuclei. In this part, we summarize the main steps of the path-integral formalism in the FK representation. Details of the calculation can 
be found in Alastuey \& Perez (1992) and Alastuey et al. (1994, 1995). The general method can be divided in two steps. In a first step only Maxwell-Boltzmann (MB) statistic is considered and all exchange effects are omitted. Using the FK representation, an equivalent classical system made of closed filaments interacting via two-body forces is introduced. Applying traditional techniques of classical mechanics inspired by Abe-Meeron's method (Meeron 1958; Abe 1959), a formal diagrammatic representation of the MB quantities was obtained, which is term-by-term well-behaved.

In a second step of this approach, the exchange contribution was introduced, evaluated perturbatively via Slater sums. Use of the FK representation leads to the appearance of open filaments (impurities) immersed in a bath of closed filaments described by MB statistics. This problem can be dealt with using the method that is explained in the MB case to inhomogeneous situations.

Collecting all the contributions arising from both the MB and the exchange treatment, a systematic virial expansion of the equation of state was obtained, which includes screening, diffraction, bound states, and exchange contributions to the ideal gas (depending on the statistics of the particles). The expression of the pressure was calculated up to and including order $\rho^{5 / 2}$ (with $\rho$ being the overall density of the system). To this order of density, the calculation of Alastuey \& Perez (1992) and Alastuey et al. $(1994,1995)$ describes exactly all the effects pertaining to bound states, diffusive and exchange processes of a system consisting of two species of elementary particles.

\subsection{Exact expression of the equation of state to the order of $\rho^{5 / 2}$}

The virial expansion of the pressure to order $\rho^{5 / 2}$ gives (Alastuey \& Perez 1992)

$$
\begin{aligned}
\beta P= & \sum_{\alpha} \rho_{\alpha}-\frac{\kappa_{D}^{3}}{24 \pi} \\
& +\frac{\pi}{6}(\ln 2-1) \sum_{\alpha, \beta} \beta^{3} e_{\alpha}^{3} e_{\beta}^{3} \rho_{\alpha} \rho_{\beta} \\
& -\frac{\pi}{\sqrt{2}} \sum_{\alpha, \beta} \rho_{\alpha} \rho_{\beta} \lambda_{\alpha \beta}^{3} Q\left(x_{\alpha \beta}\right)-\frac{\pi}{3} \beta^{3} \sum_{\alpha, \beta} \rho_{\alpha} \rho_{\beta} e_{\alpha}^{3} e_{\beta}^{3} \ln \left(\kappa_{D} \lambda_{\alpha \beta}\right) \\
& +\frac{\pi}{\sqrt{2}} \sum_{\alpha} \frac{(-1)^{2 \sigma_{\alpha}+1}}{\left(2 \sigma_{\alpha}+1\right)} \lambda^{3}{ }_{\alpha \alpha} \rho_{\alpha}^{2} E\left(x_{\alpha \alpha}\right) \\
& -\frac{3 \pi}{2 \sqrt{2}} \beta \sum_{\alpha, \beta} e_{\alpha} e_{\beta} \kappa_{D} \rho_{\alpha} \rho_{\beta} \lambda_{\alpha \beta}^{3} Q\left(x_{\alpha \beta}\right) \\
& -\frac{\pi}{2} \beta^{4} \sum_{\alpha, \beta} \rho_{\alpha} \rho_{\beta} e_{\alpha}^{4} e_{\beta}^{4} \kappa_{D} \ln \left(\kappa_{D} \lambda_{\alpha \beta}\right) \\
& +\frac{3 \pi}{2 \sqrt{2}} \beta \sum_{\alpha} \frac{(-1)^{2 \sigma_{\alpha}+1}}{\left(2 \sigma_{\alpha}+1\right)} \lambda_{\alpha \alpha}^{3} \rho_{\alpha}^{2} e_{\alpha}^{2} \kappa_{D} E\left(x_{\alpha \alpha}\right) \\
& +\frac{1}{16} \sum_{\alpha} \frac{\beta^{2} \hbar^{2} e_{\alpha}^{2}}{m_{\alpha}} \kappa_{D}^{3} \rho_{\alpha}+\pi\left(\frac{1}{3}-\frac{3}{4} \ln 2+\frac{1}{2} \ln 3\right) \\
& \times \sum_{\alpha, \beta} \beta^{4} e_{\alpha}^{4} e_{\beta}^{4} \kappa_{D} \rho_{\alpha} \rho_{\beta} \\
& +C_{1} \sum_{\alpha, \beta, \gamma} \beta^{5} e_{\alpha}^{3} e_{\beta}^{4} e_{\gamma}^{3} \kappa_{D}^{-1} \rho_{\alpha} \rho_{\beta} \rho_{\gamma} \\
& +C_{2} \sum_{\alpha, \beta, \gamma, \delta} \beta^{6} e_{\alpha}^{3} e_{\beta}^{3} e_{\gamma}^{3} e_{\delta}^{3} \kappa_{D}^{-3} \rho_{\alpha} \rho_{\beta} \rho_{\gamma} \rho_{\delta} \\
& \\
&
\end{aligned}
$$

with $\kappa_{D}=\left(4 \pi \beta \sum_{\alpha} e_{\alpha}^{2} \rho_{\alpha}\right)^{1 / 2}, \lambda_{\alpha \beta}=\left(\beta \hbar^{2} / m_{\alpha \beta}\right)^{1 / 2}, m_{\alpha \beta}=$ $m_{\alpha} m_{\beta} /\left(m_{\alpha}+m_{\beta}\right), x_{\alpha \beta}=-\sqrt{2} l_{\alpha \beta} / \lambda_{\alpha \beta}, l_{\alpha \beta}=\beta e_{\alpha} e_{\beta}, C_{1}=$ $15.205 \pm .001, C_{2}=-14.733 \pm .001$, and the Euler-Mascheroni constant $C=0.577216$.

In Eq. (14), $Q\left(x_{\alpha \beta}\right)$ refers to the so-called quantum secondvirial coefficient first introduced by Ebeling and co-workers (Ebeling et al. 1976; Kraeft et al. 1986).

$$
\begin{aligned}
& Q\left(x_{\alpha \beta}\right)= \\
& \frac{1}{\left(\sqrt{2} \pi \lambda_{\alpha \beta}^{3}\right)} \lim _{R \rightarrow \infty}\left\{\int _ { r < R } \mathrm { d } \boldsymbol { r } \left[\left(2 \pi \lambda_{\alpha \beta}^{2}\right)^{3 / 2}\left\langle\boldsymbol{r}\left|\mathrm{e}^{-\beta h_{\alpha \beta}}\right| \boldsymbol{r}\right\rangle-1\right.\right. \\
& \left.\left.+\frac{\beta e_{\alpha} e_{\beta}}{r}-\frac{\beta^{2} e_{\alpha}^{2} e_{\beta}^{2}}{2 r^{2}}\right]+\frac{2 \pi}{3} \beta^{3} e_{\alpha}^{3} e_{\beta}^{3}\left[\ln \left(\frac{3 \sqrt{2} R}{\lambda_{\alpha \beta}}\right)+C\right]\right\}
\end{aligned}
$$

while $E\left(x_{\alpha \alpha}\right)$ is defined as the two-body exchange integral,

$E\left(x_{\alpha \alpha}\right)=(2 \sqrt{\pi}) \lim _{R \rightarrow \infty} \int_{r<R} \mathrm{~d} \boldsymbol{r}\left\langle-\boldsymbol{r}\left|\mathrm{e}^{-\beta h_{\alpha \alpha}}\right| \boldsymbol{r}\right\rangle$.

In Eqs. (15) and (16), $h_{\alpha \beta}$ is the one-body Hamiltonian of the relative particle with mass $m_{\alpha \beta}$ submitted to the Coulomb potential $e_{\alpha} e_{\beta} / r$. The functions $Q$ and $E$ only depend on the temperature via the single dimensionless parameter $(-l / \lambda)$.

\subsection{Deriving other thermodynamic variables}

The expression for the pressure in Eq. (14) can be used to derive other thermodynamic variables. For example, the relationship between the pressure and the free energy is given by

$\beta P=\sum_{\alpha} \rho_{\alpha} \frac{\partial(\beta f)}{\partial \rho_{\alpha}}-\beta f$,

where $f$ is the free energy per unit volume. The internal energy per unit volume $u$ is then found as a derivative of the free energy

$u=\frac{\partial}{\partial \beta}(\beta f)_{\rho}$.

Other quantities of interest can be derived in a similar way via the usual thermodynamic derivatives.

\subsection{The general structure of $\beta P$}

First, the classical contributions of the long-range part of the interactions are polynomials in the inverse temperature, the charges and the densities, which do not involve Planck's constant. The involved coefficients are evaluated analytically or, like $C_{1}$ and $C_{2}$, by numerical computations of dimensionless integrals. The lower-order contribution is nothing but the familiar DebyeHückel term in $\rho^{3 / 2}$, and constitutes the leading correction to the MB ideal pressure in Eq. (17). It can be quoted, here, that the classical OCP limit up to order $\rho^{5 / 2}$ included does coincide with those calculated by Cohen \& Murphy (1969), as it should.

The contribution of quantum diffraction at large distances appears only at the order $\rho^{5 / 2}$ and reduces to $\frac{1}{16} \sum_{\alpha} \frac{\beta^{2} \hbar^{2} e_{\alpha}^{2}}{m_{\alpha}} \kappa_{D}^{3} \rho_{\alpha}$ The occurrence of this term shows that the long-range part of the interactions cannot be entirely treated at a classical level. The diffraction correction is merely proportional to $\hbar$, because it arises from large distances where the quantum effects can be treated perturbatively "à la-Wigner-Kirkwood”. 
The term $\rho_{\alpha} \rho_{\beta} \lambda_{\alpha \beta}^{3} Q\left(-\sqrt{2} \frac{l_{\alpha \alpha}}{\lambda_{\alpha \alpha}}\right)$ is the total contribution from both bound and scattering states of two charges $e_{\alpha}$ and $e_{\beta}$. The truncation of $\left\langle\boldsymbol{r}\left|\exp \left(-\beta h_{\alpha \beta}\right)\right| \boldsymbol{r}\right\rangle$ in the integral defining $Q$ ensures that this contribution is finite. This regularization is not an arbitrary mathematical artifact. It is directly related to the truncated structure of the bond $f_{T}$, and reflects the screening of the Coulomb interaction at large distances. For opposite charges such that $e_{\alpha} e_{\beta}<0$, one may extract from $Q$ a contribution of the bound states which reduces to the familiar Planck-Larkin (PL) sum

$\sum_{n=1}^{\infty} n^{2}\left[\exp \left(-\beta \epsilon_{n}^{\alpha \beta}\right)-1+\beta \epsilon_{n}^{\alpha \beta}\right]$

where $\epsilon_{n}^{\alpha \beta}=-e_{\alpha}^{2} e_{\beta}^{2} m_{\alpha \beta} /\left(2 \hbar^{2} n^{2}\right)$ are the energy levels of the hydrogenic atom with Hamiltonian $h_{\alpha \beta}$. However, other definitions of the bound states contributions can be introduced from Eq. (15) by using the basic properties of the trace. For instance, as shown by Bollé (1987), there exists an infinite set of arbitrary decompositions in terms of bound and scattering contributions of the PL sum itself. So, as far as thermodynamic quantities are concerned, only the total contribution of both bound and scattering states is an unambiguous quantity. The $\rho^{5 / 2}$-contribution from bound and scattering states merely reduces to its $\rho^{2}$ counterpart multiplied by $\beta e_{\alpha} e_{\beta} K_{D}$. This multiplicative factor arises from many-body effects which induce a constant shift $-e_{\alpha} e_{\beta} K_{D}$ on the energy levels of the two-particles states.

Finally, the contribution $\rho_{\alpha}^{2} E\left(-\sqrt{2} \frac{l_{\alpha \alpha}}{\lambda_{\alpha \alpha}}\right)$ arises from the exchange of two charges $e_{\alpha}$ in the vacuum. It is finite, independently of any screening effect, because the off-diagonal matrix elements $\left\langle-\boldsymbol{r}\left|\exp \left(-\beta h_{\alpha \alpha}\right)\right| \boldsymbol{r}\right\rangle$ are short ranged. The magnitude of this contribution is smaller than the one relative to free particles because the repulsive potential $e_{\alpha}^{2} / r$ inhibits the exchange. Similarly to what happens for the contributions of bound and scattering states, at the order $\rho^{5 / 2}$, the many-body effects on the two-particle exchange amounts to lowering the repulsive barrier $e_{\alpha}^{2} / r$ by the constant $-e_{\alpha}^{2} \kappa_{D}$.

\subsection{The nature of the non-ideal terms in the FK equation of state}

As is usual for a weakly non-ideal plasma (see, e.g. Ebeling et al. 1976), the most important contributions beyond the ideal gas limit are the exchange terms, the Debye screening corrections, the short-range corrections and diffraction (in decreasing order of importance). Let us recall briefly the various conditions allowing us to check the nature of the short-range corrections for a given temperature and density.

Since the current level of our FK equation of state is truncated at the order $\rho^{5 / 2}$, it can only include processes with two-body effects. It comes naturally, from the structure of the equation of state, that short-range quantum effects are virtually decoupled from the other corrections. This means that in all orders of density, the main impact of these short-range quantum corrections merely pertains to the recombination and ionization processes. The magnitude of the neglected higher-order terms will indicate the influence of three-body or higher interactions. The comparison with solar data will allow us a quantitative estimate of these neglected terms.

Similarly, one has to be aware of the higher Debye corrections beyond the $\rho^{5 / 2}$ term. A careful analysis of the virial expansion shows that the quantum short-range correction that only involves the two-body virial coefficient $Q\left(x_{\alpha \beta}\right)$ results in an al-

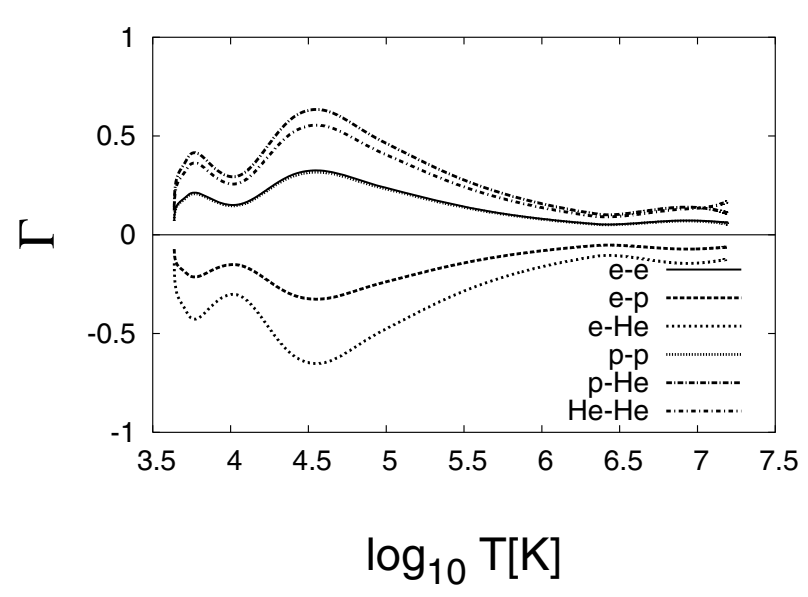

Fig. 2. Coupling parameters for electrons, protons, and helium nuclei from the surface to the center of the Sun.

ternating series in $\rho$, which is therefore convergent. Since the leading Debye correction to pressure (included in our formalism) is negative, the following term must be positive. Should at some physical conditions the neglected higher corrections lead to a lowering of pressure, we will be able to interpret them as a manifestation of a recombination process occurring under these thermodynamical conditions.

\section{Limits of validity of the FK equation of state}

\subsection{Dimensionless parameters}

A detailed analysis of this equation of state suggests rewriting the truncated expression of the thermodynamic quantities (for example pressure) in terms of three characteristic lengths:

$$
\begin{aligned}
& \text { - mean interparticle distance } d_{\alpha}=\left(3 / 4 \pi n_{\alpha}\right)^{1 / 3} ; \\
& \text { - thermal de Broglie wavelength } \Lambda_{\alpha}=\left(\beta h^{2} / m_{\alpha}\right)^{1 / 2} ; \\
& \text { - Landau length } \quad l_{\alpha \beta}=\beta e_{\alpha} e_{\beta} .
\end{aligned}
$$

Here the notation is standard, and there should be no confusion between $\beta$ being both a label and $1 /(k T)$. Note that since we are now dealing with quantitative applications, we denote number density by the usual symbol $n$ rather than the $\rho$ that was used in the preceding theoretical sections of this paper.

Before the FK equation of state can be applied to the Sun, we must determine where the formalism is applicable. Here we evaluate the relevant criteria throughout the Sun, based on a standard solar model (Model S from Christensen-Dalsgaard, as described in Christensen-Dalsgaard et al. 1996) First, we verify the wellknown result that the plasma in the solar interior is indeed not strongly coupled. Fig. 2 shows the coupling parameter

$\Gamma_{\alpha}=\frac{l_{\alpha \alpha}}{d_{\alpha}}$

through the solar model. The index $\alpha$ denotes electrons, protons, and helium nuclei, respectively. Nowhere does the coupling parameter $\Gamma_{\alpha}$ come near to the critical value of unity.

Figs. 3-5 show the degeneracy parameter $n_{\alpha} \Lambda_{\alpha}^{3}$, again for electrons, protons, and helium nuclei, respectively.

\subsection{Qualitative discussion of ionization and recombination}

As we discussed in detail in Sect. 2, avoiding too much recombination is the most restrictive criterion for the FK equation of 


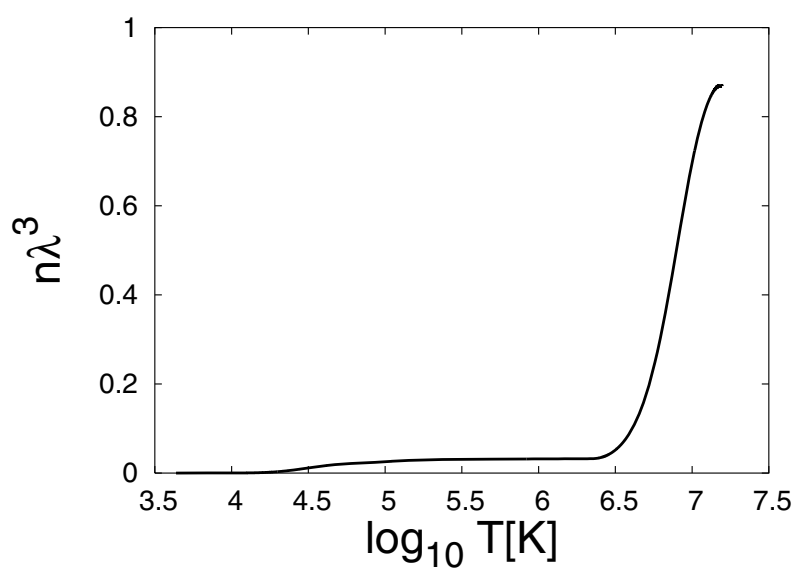

Fig. 3. Degeneracy parameter for electrons from the surface to the center of the Sun.

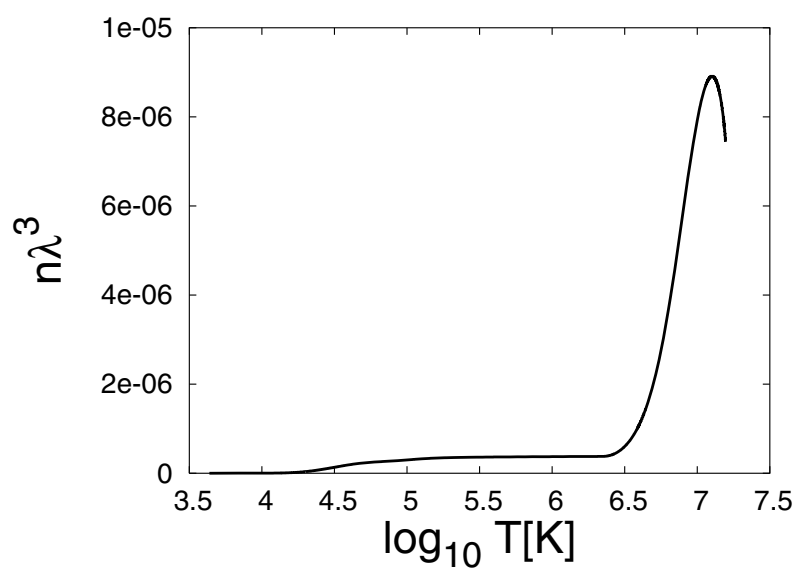

Fig. 4. Degeneracy parameter for protons from the surface to the center of the Sun.

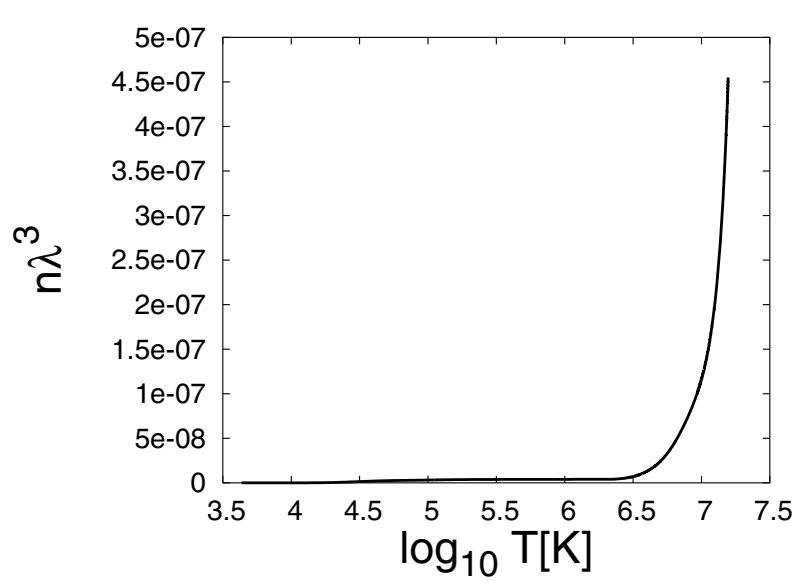

Fig. 5. Degeneracy parameter for helium nuclei from the surface to the center of the Sun.

state. Figures 6-9 show that it is certainly more restrictive than coupling and degeneracy. Obviously, our low-density virial expansion cannot be used in the cooler outer layers of the Sun, where the plasma is far from fully ionized. To obtain an estimate of limit of validity of FK, which we identified with $90 \%$ ionization, we have calculated the hydrogen and helium ionization

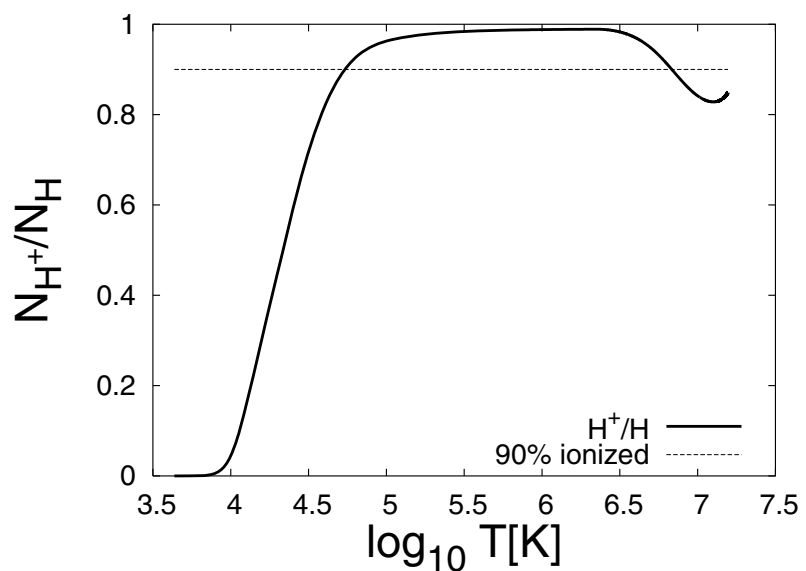

Fig. 6. Ionization degree of hydrogen from the surface to the center of the Sun.

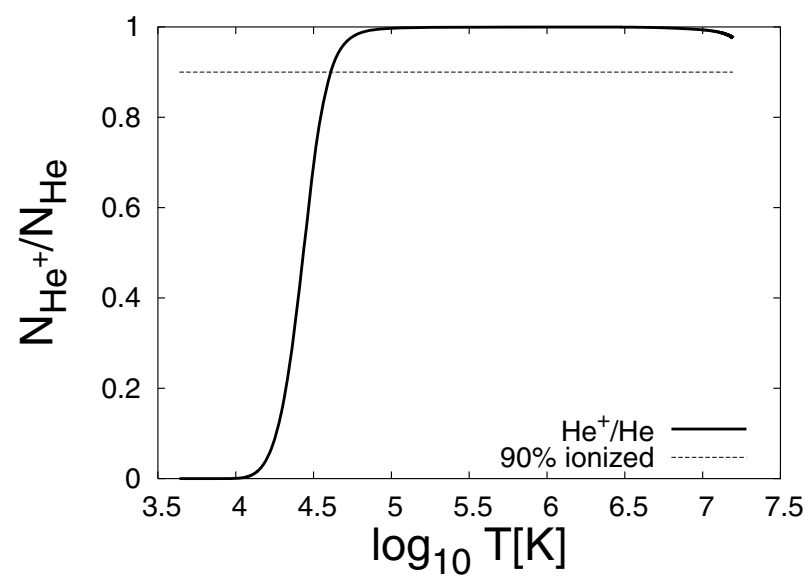

Fig. 7. First ionization degree of helium from the surface to the center of the Sun.

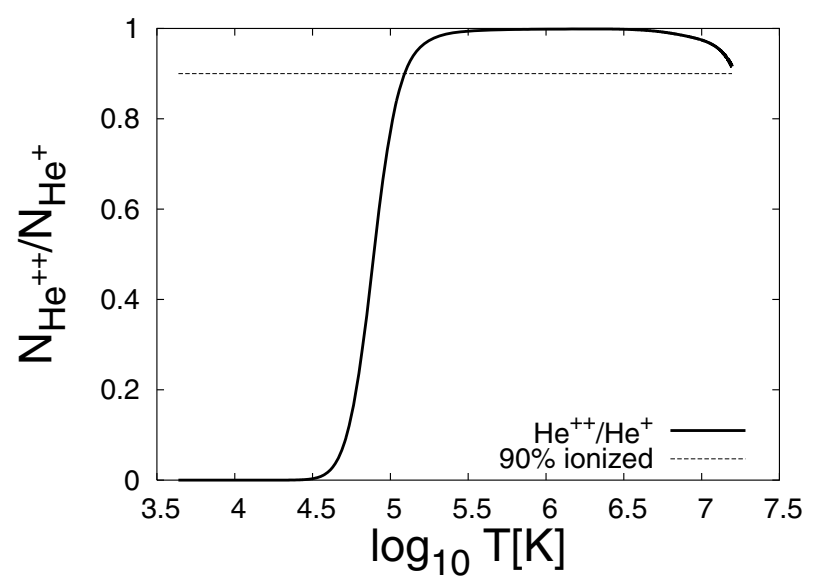

Fig. 8. Second ionization degree of helium from the surface to the center of the Sun.

fraction using the standard (ground-state-only) Saha equation

$\frac{N_{Z+1} N_{e^{-}}}{N_{Z}}=\left(\frac{g_{Z+1} g_{e^{-}}}{g_{Z}}\right) \frac{V}{\Lambda_{\alpha}^{3}} \exp \left(-\frac{\chi Z}{k T}\right)$

throughout the same solar model. As usual, $N_{\alpha}$ and $g_{\alpha}$ denote the number of particles and statistical weights of the various species (here identified by the ion charge $Z$ ) and $\chi_{Z}$ is the ionization 


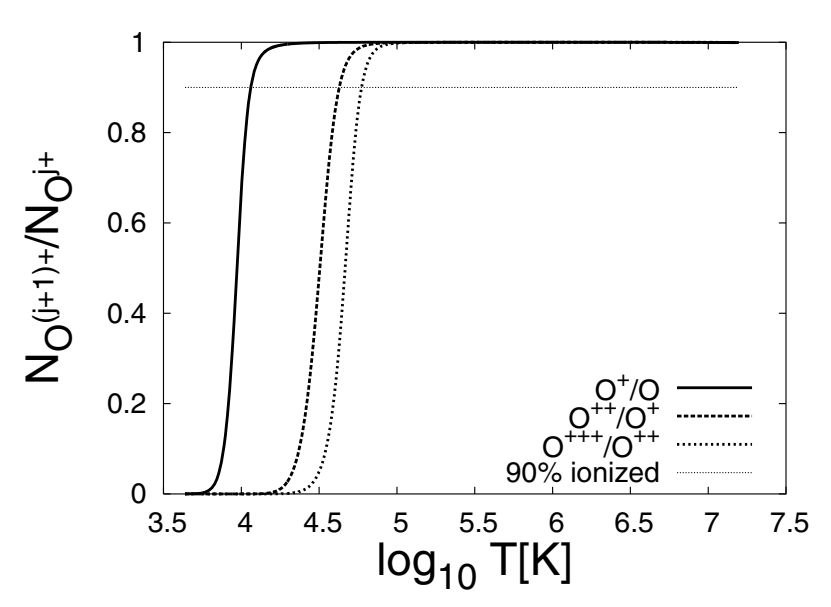

Fig. 9. First and second ionization degree of oxygen (as a representative heavy element) from the surface to the center of the Sun.

potential pertaining to the ionization reaction $Z \rightarrow Z+1$. Using this simple Saha equation for this purpose is alright since we use it only for a kind of error analysis. And, as numerous studies have shown, for solar conditions, this simple Saha equation is everywhere at most $10 \%$ wrong (e.g. Christensen-Dalsgaard \& Däppen 1992).

Figures 6-9 show the recombination fraction for hydrogen, helium, and the first two ionization stages of oxygen (as a typical representative heavy element). The $90 \%$-ionization level is also shown. Clearly, at some point in the colder outside layers, our virial expansion must break down.

However, in the deeper layers of the Sun (and non-compact stars in general), we can verify that the weak-coupling and low-degeneracy criteria are fully satisfied and the limitation from the "recombination criterion" is totally negligible for the temperatures and densities of those regions.

\subsection{The treatment of heavier elements}

Adding heavier elements (other than hydrogen and helium) reduces the domain of validity of the FK equation of state to even higher temperatures. This is obvious because it is harder to ionize these elements. Figure 9 shows this quantitatively for oxygen. We therefore adopt the simplest solution which is to ignore the heavier elements altogether. Indeed, in the solar application of Paper II, we will merely add the pressure of the heavier elements to the FK expansion 14. Since in the Sun, the number abundance of all elements other than hydrogen and helium is only about $10^{-3}$, adding the heavier elements to FK with an equation of state such as MHD, which is at least as accurate as $1 \%$ (e.g. Däppen 2006) will limit the total error of such a simplified procedure to an acceptable order of magnitude of $10^{-5}$.

In addition, knowing the domain of validity also allows us to study selected recombination reactions in the framework of the FK equation of state. For instance, in the calculation of nuclear reaction cross sections, the ionization degree of the various nuclei plays a role, because the statistical presence of electrons around the nucleus modulates the reaction rate (enhancement factor). This has consequences for solar physics especially with respect to the neutrino problem, where the reactions involving $\mathrm{Be}$ and $\mathrm{B}$ have to be know very accurately (Perez \& Chabrier 2004).

\subsection{Solar models with a hybrid equation of state}

Because of its limitations to nearly full ionization, it is impossible to use the FK formalism for the entire Sun. Therefore, for our applications in Paper II, we will have to use a hybrid two-zone equation of state, consisting of FK serving in the interior, and a more conventional one (OPAL or MHD) in the outer layers. Although such a hybrid equation of state will have discontinuities at the transition, these discontinuities will not preclude the solution of the usual stellar structure equations. Their main effect is expected to be a small jump in the gradient of the physical quantities, similar to that caused, for instance, by standard interpolation in equation-of-state and opacity tables. As detailed studies (Christensen-Dalsgaard \& Däppen 1992) have shown, successful equation of state tests can still be made despite such small imperfections. The analysis of Paper II will reveal if the small discontinuities will cause problems. If so, they can be avoided by the introduction of suitable smooth ramp functions at the juncture line of the two-zone equation of state.

\subsection{Distribution of FK equation of state}

OPAL and MHD have been made available to solar and stellar modelers through the use of tables. Although this saves the modelers a bit of computing time, the tables have only been provided for a limited range of conditions. In order to make the FK equation of state more versatile, we plan to develop a computational routine for which users can define their own input parameters such as element abundances. This implementation of FK will be ideally suited for exploring issues such as He diffusion and the inconsistency between seismology and the new values of heavy element abundances (Asplund 2005; Asplund et al. 2005; Grevesse et al. 2007).

\section{Conclusions}

Despite the limitation to nearly fully ionized matter, the FK approach can be applied to large parts of the solar interior, more precisely to the part where helium is at least $90 \%$ ionized. In those regions the FK approach is applicable, and it is especially suited to study screening effects, bound states, recombination of fully ionized ions, and exchange effects.

Paper II will be dedicated to these observational tests. Since the important relativistic correction for electrons (Elliott \& Kosovichev 1998) is not part of the original FK equation of state (14), we will add it in Paper II. Paper III will go further, on a rigorous theoretical level, to the calculation of the thermodynamic functions up to order $\rho^{3}$ in density. The additional higherorder terms will allow us to take into account the three-body effects that occur in a moderately ionized plasma (for example, in the case of the solar center, the possibility of a recombination of $\mathrm{He}^{++}$is still an unsolved problem).

Despite the severe technical difficulties involving the practical application of the FK equation of state for solar physics, there are unique features in the FK approach that promise to turn it into the most exact of the available formalisms, if they are limited to the layers of the Sun where more than $90 \%$ of helium is ionized. The localizing power of helioseismology allows us to test FK observationally, promising both better and tighter constraints on the equation of state. For instance, by virtue of its exactness, the FK equation of state becomes a promising tool for tweaking parameterizable equations of state such as MHD. More generally, the FK equation of state will lead to better solar models as well as tighter solar constraints of the equation of state. 
Acknowledgements. We thank Jørgen Christensen-Dalsgaard for the solar model used in this study. This work was supported by the grant AST-0708568 of the National Science Foundation.

\section{References}

Abe, R., 1959, Progr. Theor. Phys., 22, 213

Alastuey, A., \& Perez, A. 1992, Europhys. Lett., 20, 19

Alastuey, A., \& Perez, A. 1996, Phys. Rev. E, 53, 5714

Alastuey, A., Cornu, F., \& Perez, A. 1994, Phys. Rev. E, 49, 1077

Alastuey, A., Cornu, F., \& Perez, A. 1995, Phys. Rev. E, 51, 1725

Asplund, M. 2005, ARA\&A, 43, 481

Asplund, M. Grevesse, N., \& Sauval, A. J. 2005, in Cosmic Abundances as Records of Stellar Evolution and Nucleosynthesis, San Francisco, California, ed. T. G. Barnes III, \& F. N. Bash, ASP Conf. Ser., 336, 25

Berrington, K. A. 1997, The Opacity Project, Vol. II (Bristol: Institute of Physics Publishing)

Bollé, D. 1987, Phys. Rev. A, 36, 3259

Christensen-Dalsgaard, J., \& Däppen, W. 1992, A\&A, 4, 267

Christensen-Dalsgaard, J., Däppen, W., \& the GONG Team 1996, Science, 272, 1286

Cohen, E. G. D., \& Murphy, T. J. 1969, Phys. Fluids, 12, 1404

Däppen, W. 2006, J. Phys. A: Math. Gen., 39, 4441

Däppen, W., \& Mao, D. 2009, J. Phys. A: Math. Theor., 42, 214006

Däppen, W., Mihalas, D., Hummer, D. G., \& Mihalas, B. W. 1988, ApJ, 332, 261

Ebeling, W., Kraeft, W. D., Kremp, D., 1976, Theory of Bound States and Ionization Equilibrium in Plasmas and Solids (Berlin, DDR: Akademie Verlag)

Eggleton, P. P., Faulkner, J., \& Flannery, B. P. 1973, A\&A, 23, 325

Elliott, J. R., \& Kosovichev, A. G. 1998, ApJ, 500, L199

Grevesse, N., Asplund, M. \& Sauval, A. J. 2007, Space Sci. Rev., 130, 105

Huang K. 1963, Statistical Mechanics (New York: John Wiley), Chapt. 14
Hummer, D. G., \& Mihalas, D. 1988, ApJ, 331, 794

Iglesias, C. A., Rogers, F. J., \& Wilson, B. G. 1987, ApJ, 322, L45

Iglesias, C. A., \& Rogers, F. J., 1991, ApJ, 371, 408

Iglesias, C. A., \& Rogers, F. J. 1993, ApJ, 412, 752

Iglesias, C. A., \& Rogers, F. J. 1995, ApJ, 443, 460

Iglesias, C. A., \& Rogers, F. J. 1996, ApJ, 464, 943

Kraeft W. D., Kremp D., Ebeling W., Röpke G., 1986, Quantum Statistics of Charged Particle Systems (New York: Plenum)

Kremp D., Schlanges, M., Kraeft, W.-D., 2005, Quantum Statistics of Nonideal Plasmas (Springer: Berlin and Heidelberg)

Liang, A., 2004, in Equation-of-State and Phase-Transition Issues in Models of Ordinary Astrophysical Matter, ed. V. Celebonovic, W. Däppen, \& D. Gough, (New York: Melville), AIP Conf. Proc., 731, 106

Liang, A., \& Däppen, W. 2003, in Proc. SOHO 12/GONG+ 2002 Workshop (Noordwijk, The Netherlands), ESA SP-517, 333

Meeron, E., 1958, J. Chem. Phys., 28, 630

Mihalas, D., Däppen W., \& Hummer, D. G. 1988, ApJ, 331, 815

Nayfonov, A., Däppen, W., Hummer, D. G., \& Mihalas, D. M. 1999, ApJ, 526, 451

Perez, A., \& Chabrier, G. 2004, in Equation-of-State and Phase-Transition Issues in Models of Ordinary Astrophysical Matter, ed. V. Celebonovic, W. Däppen, \& D. Gough, (New York: Melville), AIP Conf. Proc., 731, 208

Perez, A., \& Däppen, W. 1995, in Proc. of Fourth SOHO Workshop Helioseismology (June 1995), ESA-SP-376, 15

Rogers, F.J. 1986, ApJ, 310, 723

Rogers, F. J., \& Nayfonov, A. 2002, ApJ, 576, 1064

Rogers, F. J., Swenson, F. J., \& Iglesias, C. A. 1996, ApJ, 456, 902

Seaton, M. J. 1995, The Opacity Project, Vol. I (Bristol: Institute of Physics Publishing)

Starostin A., Roerich, V. C., \& More, R. M. 2003, Contrib. Plasma Phys., 43, 369

Trampedach, R., Däppen, W., \& Baturin, V. A., 2006, ApJ, 646, 560 